\title{
ON THE STABLE RANK AND REDUCIBILITY IN ALGEBRAS OF REAL SYMMETRIC FUNCTIONS
}

\author{
R. RUPP AND A. SASANE
}

\begin{abstract}
Let $A_{\mathbb{R}}(\overline{\mathbb{D}})$ denote the set of functions belonging to the disc algebra having real Fourier coefficients. We show that $A_{\mathbb{R}}(\overline{\mathbb{D}})$ has Bass and topological stable ranks equal to 2 , which settles the conjecture made by Brett Wick in [18]. We also give a necessary and sufficient condition for reducibility in some real algebras of functions on symmetric domains with holes, which is a generalization of the main theorem in [18]. A sufficient topological condition on the symmetric open set $D$ is given for the corresponding real algebra $A_{\mathbb{R}}(\bar{D})$ to have Bass stable rank equal to 1.
\end{abstract}

\section{CDAM Research Report LSE-CDAM-2007-20}

\section{INTRODUCTION}

The notion of stable rank of a ring (which we call Bass stable rank) was introduced by H. Bass [2] to facilitate computations in algebraic K-theory. We recall the definition of the Bass stable rank of a ring below.

Definition 1.1. Let $\mathcal{A}$ be a commutative ring with identity 1 . Let $n \in \mathbb{N}$. An element $a=\left(a_{1}, \ldots, a_{n}\right) \in \mathcal{A}^{n}$ is called unimodular if there exists $b=\left(b_{1}, \ldots, b_{n}\right) \in \mathcal{A}^{n}$ such that

$$
\langle b, a\rangle:=\sum_{k=1}^{n} b_{k} a_{k}=1
$$

We denote by $U_{n}(\mathcal{A})$ the set of unimodular elements of $\mathcal{A}^{n}$.

We say that $a=\left(a_{1}, \ldots, a_{n}\right) \in U_{n}(\mathcal{A})$ is reducible (in $\mathcal{A}$ ), if there exist $h_{1}, \ldots, h_{n-1} \in \mathcal{A}$ such that $\left(a_{1}+h_{1} a_{n}, \ldots, a_{n-1}+h_{n-1} a_{n}\right) \in U_{n-1}(\mathcal{A})$. The Bass stable rank of $\mathcal{A}$, denoted by bsr $\mathcal{A}$, is the least integer $n$ such that every $a \in U_{n+1}(\mathcal{A})$ is reducible (and it is infinite if no such integer $n$ exists).

The Bass stable rank is a purely algebraic notion, but when studying commutative Banach algebras of functions, analysis also plays a role. In [13], M. Rieffel introduced the notion of topological stable rank, analogous to the concept of Bass stable rank:

Definition 1.2. Let $\mathcal{A}$ denote a commutative unital Banach algebra. The topological stable rank of $\mathcal{A}$, denoted by tsr $\mathcal{A}$, is the minimum integer $n$ such that $U_{n}(\mathcal{A})$ is dense in $\mathcal{A}^{n}$ (and it is infinite if no such integer exists).

1991 Mathematics Subject Classification. Primary 46J15; Secondary 19B10, 30H05, 93D15.

Key words and phrases. Real Banach algebras, Bass stable rank, topological stable rank, reducibility, stabilization.

Part of this work was done while R. Rupp visited London School of Economics. He wishes to thank LSE for their kind hospitality and the University of Applied Sciences, Nürnberg for the sabbatical.

A. Sasane is supported by the Nuffield Grant NAL/32420. 
Jones, Marshall and Wolff [9] showed that the Bass stable rank of the complex disc algebra $A(\overline{\mathbb{D}})$ is equal to 1 , and Rieffel [13] showed that its topological stable rank is equal to 2 . Recall that the complex disc algebra $A(\overline{\mathbb{D}})$ is the Banach algebra of all complex-valued functions defined on the closed unit disc $\overline{\mathbb{D}}$ that are holomorphic in the open unit disc $\mathbb{D}$ and continuous on $\overline{\mathbb{D}}$, endowed with the supremum norm: $\|f\|_{\infty}=\sup _{z \in \overline{\mathbb{D}}}|f(z)|$.

In this article, we study the Bass/topological stable ranks, and also consider reducibility of corona pairs, in some real Banach algebras of "real symmetric" functions. We define these below. Throughout this article, we use $z^{*}$ to denote the complex conjugate of $z$, and we use $\bar{\Omega}$ to denote the closure of the set $\Omega \subset \mathbb{C}$.

Definition 1.3. The real disc algebra, denoted by $A_{\mathbb{R}}(\overline{\mathbb{D}})$, is the set of all functions of $A(\overline{\mathbb{D}})$ having real Fourier coefficients. Equivalently,

$$
A_{\mathbb{R}}(\overline{\mathbb{D}})=\left\{f \in A(\overline{\mathbb{D}}) \mid \forall z \in \overline{\mathbb{D}}, f(z)=\left(f\left(z^{*}\right)\right)^{*}\right\} .
$$

The real disc algebra $A_{\mathbb{R}}(\overline{\mathbb{D}})$ is a real Banach algebra with the supremum norm $\|\cdot\|_{\infty}$.

More generally, if $D$ is an open set in $\mathbb{C}$, then by $A(\bar{D})$ we mean the set of functions holomorphic in $D$ that are continuous and bounded on $\bar{D}$. If $D$ is real symmetric (that is, $z \in D$ if and only if $\left.z^{*} \in D\right)$, then we use the symbol $A_{\mathbb{R}}(\bar{D})$ to denote the set of functions $f$ belonging to $A(\bar{D})$ that are real symmetric, that is, $f(z)=\left(f\left(z^{*}\right)\right)^{*}(z \in \bar{D})$.

If $D$ is a real symmetric open set, then $C_{\mathbb{R}}(\bar{D})$ denotes the set of complex-valued, bounded, continuous functions $f$ defined on $\bar{D}$, that satisfy $f(z)=\left(f\left(z^{*}\right)\right)^{*}(z \in \bar{D})$.

Brett Wick conjectured [18] that the Bass stable rank of $A_{\mathbb{R}}(\overline{\mathbb{D}})$ is equal to 2 , and we prove this in Section 2, by first showing that the topological stable rank of $A_{\mathbb{R}}(\overline{\mathbb{D}})$ is 2 . In Section 4 we extend the main result of $\mathrm{B}$. Wick [18] to the case of subalgebras of $A_{\mathbb{R}}(\overline{\mathbb{D}})$. We also completely characterize reducible elements in algebras $A_{\mathbb{R}}(\bar{D})$ of real symmetric functions on domains with holes (under mild assumptions) in Theorem 6.4. This generalizes the main result in [18] from the case of the disc to more general domains. Finally, in Theorem 6.6, we give a sufficient topological condition on the open set $D$ for $A_{\mathbb{R}}(\bar{D})$ to have Bass stable rank equal to 1 .

\section{Bass AND topological Stable RANK of $A_{\mathbb{R}}(\overline{\mathbb{D}})$}

In this section we prove that bsr $A_{\mathbb{R}}(\overline{\mathbb{D}})=\operatorname{tsr} A_{\mathbb{R}}(\overline{\mathbb{D}})=2$.

We begin by making the observation that the polynomials with real coefficients are dense in $A_{\mathbb{R}}(\overline{\mathbb{D}})$. Indeed, given $f \in A_{\mathbb{R}}(\overline{\mathbb{D}}), f$ has real Fourier coefficients, which are the same as the coefficients in the Taylor expansion of the analytic function $f$ about the point 0 in $\mathbb{D}$. Since $f$ is continuous on the circle, and its negative Fourier coefficients vanish, the Cesàro means of the Fourier series for $f$ are trigonometric polynomials with real coefficients which converge uniformly to $f$. The corresponding polynomials in $z$ give the desired sequence converging uniformly to $f$ in $A_{\mathbb{R}}(\overline{\mathbb{D}})$.

Theorem 2.1. The topological stable rank of $A_{\mathbb{R}}(\overline{\mathbb{D}})$ is 2 .

Proof. First of all we note that $U_{1}\left(A_{\mathbb{R}}(\overline{\mathbb{D}})\right)$ is not dense in $A_{\mathbb{R}}(\overline{\mathbb{D}})$. Indeed, $U_{1}\left(A_{\mathbb{R}}(\overline{\mathbb{D}})\right)$ is the set of units in $A_{\mathbb{R}}(\overline{\mathbb{D}})$, and $f$ is invertible as an element in $A_{\mathbb{R}}(\overline{\mathbb{D}})$ only if it has no zero in $\overline{\mathbb{D}}$. But the uniform limit of a sequence of functions from the disc algebra which are never zero in $\mathbb{D}$ is either identically zero or has no zeros in $\mathbb{D}$; see [1, Theorem 2, p. 178]. So taking any function with finitely many zeros in $\mathbb{D}$, say $z$, we have a contradiction. So tsr $A_{\mathbb{R}}(\overline{\mathbb{D}})>1$. 
Next we show that $U_{2}\left(A_{\mathbb{R}}(\overline{\mathbb{D}})\right)$ is dense in $A_{\mathbb{R}}(\overline{\mathbb{D}})^{2}$. Take $(f, g) \in A_{\mathbb{R}}(\overline{\mathbb{D}})^{2}$ and approximate $f, g$ by polynomials $p, q$, respectively, having real coefficients. Since $p \in \mathbb{R}[z]$, we have the following product representation for $p$ :

$$
p(z)=C \prod\left(z-r_{j}\right) \prod\left(z^{2}+s_{j} z+t_{j}\right),
$$

where $C, r_{j}, s_{j}, t_{j}$ are real numbers. If $p$ and $q$ have a common root in $\overline{\mathbb{D}}$, then we replace $r_{j}, s_{j}, t_{j}$ by $r_{j}+\epsilon, s_{j}+\epsilon, t_{j}+\epsilon$ with a sufficiently small real $\epsilon$ so that the new polynomial $\widetilde{p}$ has no common root with $q$ in $\overline{\mathbb{D}}$, and so $(\widetilde{p}, q) \in U_{2}\left(A_{\mathbb{R}}(\overline{\mathbb{D}})\right)$ is near $(f, g)$. Consequently tsr $A_{\mathbb{R}}(\overline{\mathbb{D}}) \leq 2$.

We recall the following result [4, Theorem 3, p. 293]:

Proposition 2.2. Let $\mathcal{A}$ be a commutative unital real (or complex) Banach algebra. If $U_{n}(\mathcal{A})$ is a dense subset of $\mathcal{A}^{n}$, then $\operatorname{bsr} \mathcal{A} \leq n$.

Theorem 2.3. The Bass stable rank of $A_{\mathbb{R}}(\overline{\mathbb{D}})$ is 2 .

Proof. First we show that bsr $A_{\mathbb{R}}(\overline{\mathbb{D}})>1$. Consider $a:=\left(z, 1-z^{2}\right) \in A_{\mathbb{R}}(\overline{\mathbb{D}})^{2}$. The element $a$ is unimodular, since with $b:=(z, 1) \in A_{\mathbb{R}}(\overline{\mathbb{D}})^{2}$, we have $\langle b, a\rangle=1$. However $a$ is not reducible. Indeed, otherwise there exists an element $h \in A_{\mathbb{R}}(\overline{\mathbb{D}})$ such that with $f(z):=z+h(z)\left(1-z^{2}\right)$, $z \in \overline{\mathbb{D}}, f$ is an invertible element of $A_{\mathbb{R}}(\overline{\mathbb{D}})$. However, $f(-1)=-1$ and $f(1)=1$, and so by the intermediate value theorem $f(c)=0$ for some $c \in(-1,1)$, contradicting the invertibility of $f$.

From Theorem 2.1 it follows that $U_{2}\left(A_{\mathbb{R}}(\overline{\mathbb{D}})\right)$ is dense in $A_{\mathbb{R}}(\overline{\mathbb{D}})^{2}$, and so by Proposition 2.2 , we obtain that bsr $A_{\mathbb{R}}(\overline{\mathbb{D}}) \leq 2$. This completes the proof.

\section{Remark 2.4.}

(1) Brett Wick conjectured in [18] that the Bass stable rank of $A_{\mathbb{R}}(\overline{\mathbb{D}})$ is equal to 2; the above result settles this conjecture.

(2) Bass and topological stable ranks of $A_{\mathbb{R}}(\overline{\mathbb{D}})$ play an important role in control theory in the problem of stabilization of linear systems. We refer the reader to [12] and [17] for background on the connection between stable rank and control theory.

\section{Preliminaries}

3.1. Reducibility in general real Banach algebras. We adapt the definition from [14, Definition 1.1] to the case of real Banach algebras as follows:

Definition 3.1. Let $\mathcal{A}$ be a real commutative Banach algebra with unit element denoted by 1. Suppose that $(f, g) \in \mathcal{A}^{2}$ and $\lambda, \mu \in \mathbb{R}$. The real numbers $\lambda, \mu$ are called equivalent if there exists elements $h, k \in \mathcal{A}$ such that

$$
f-\lambda+h g=(f-\mu) \exp (k)
$$

It is not hard to see that for fixed $(f, g)$ this indeed gives an equivalence relation. We denote the equivalence class of $\lambda$ by $[\lambda]$.

Theorem 3.2. If $(f-\mu, g) \in U_{2}(\mathcal{A})$, then $[\mu]$ is open in $\mathbb{R}$.

The proof is the same as that of [14, Proposition 1.2], but all numbers have to be real. 
Theorem 3.3. Let $(f, g) \in U_{2}(\mathcal{A})$ and suppose that for some positive $\epsilon$ the interval $(-\infty, \epsilon)$ belongs to the real inversion set

$$
I_{\mathbb{R}}(f, g):=\left\{\lambda \in \mathbb{R} \mid(f-\lambda, g) \in U_{2}(\mathcal{A})\right\} .
$$

Then $(f, g)$ is reducible in $\mathcal{A}$.

The proof is similar to that of [14, Proposition 1.3]: Take a real number $M$ such that $M>\|f\|$. Then there exists $l \in \mathcal{A}$ such that $f-(-M)=\exp (l)$. Then $(f-(-M), g) \in U_{2}(\mathcal{A})$ and, by assumption, $-M$ and 0 belong to the same connected component of $I_{\mathbb{R}}(f, g)$. But then Theorem 3.2 implies that $\mu=-M$ and $\lambda=0$ are equivalent. (Otherwise the open connected set $(-\infty, \epsilon)$ would split into disjoint open sets, namely certain equivalence classes.) Thus there exist $h, k \in \mathcal{A}$ such that $f+h g=(f-(-M)) \exp (k)=\exp (k+l)$.

3.2. Some notation and terminology. When we consider domains with holes in sections 5 and 6 , the following notation will be convenient.

Notation 3.4. Let $D$ denote a bounded symmetric domain in $\mathbb{C}$ with $n$ holes, having a boundary that is a union of pairwise disjoint Jordan curves. The outer boundary curve is denoted by $\Gamma_{n+1}$. From these $n$ holes, bounded by pairwise disjoint Jordan curves $\Gamma_{j}$, we have $r$ holes intersecting $\mathbb{R}$ and $2 m$ which do not intersect $\mathbb{R}$. Here $n=r+2 m$. For notational reasons the Jordan curves $\Gamma_{j}$ belonging to the upper half plane are indexed by $j=r+1, \ldots, r+m$, while the Jordan curves belonging to the lower half plane are indexed by $j=r+m+1, \ldots, r+2 m$. These curves $\Gamma_{r+m+j}$ are the reflection of $\Gamma_{r+j}$. Let $C_{j}$ be a hole of $D(j=1, \ldots, r, r+1, \ldots, r+2 m)$. Choose for each $j \in\{1, \ldots, r\}$ a point $x_{j} \in \mathbb{R} \cap C_{j}$, and for $j \in\{r+1, \cdots r+m\}$ let $z_{j} \in C_{j}$. Finally let $S=\left\{x_{1}, \ldots, x_{r}, z_{1}, \ldots, z_{m}, z_{1}^{*}, \ldots, z_{m}^{*}\right\}$. See Figure 1. To ensure that the domain $D$ is always to the left of the orientation, we assume that the Jordan curves $\Gamma_{1}, \ldots, \Gamma_{n}$ surrounding the holes are negatively oriented, whereas the outer boundary curve $\Gamma_{n+1}$ is positively oriented.

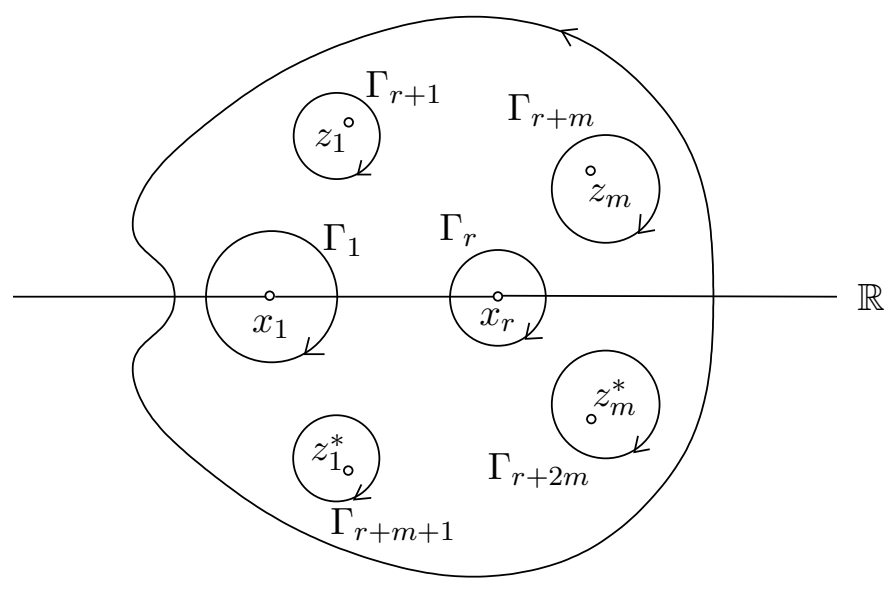

FiguRE 1 . The domain $D$ with $n=r+2 m$ holes.

Definition 3.5. If $D$ denotes an open subset of $\mathbb{C}$, then we say the corona theorem holds for $\mathcal{A}\left(\subset A_{\mathbb{R}}(\bar{D})\right)$ if the following is true for all $n \in \mathbb{N}$ :

$\left(f_{1}, \ldots, f_{n}\right) \in U_{n}(\mathcal{A})$ if and only if there exists $\delta>0$ such that $\sum_{j=1}^{n}\left|f_{j}(z)\right| \geq \delta(z \in \bar{D})$, that is, if and only if the functions $f_{1}, \ldots, f_{n}$ have no common zero in $\bar{D}$. 
Definition 3.6. For functions $g \in A_{\mathbb{R}}(\bar{D})$ the zero set $Z_{g}$ of $g$ is

$$
Z_{g}:=\{z \in \bar{D} \mid g(z)=0\},
$$

and for $\delta>0$ the level set $Z(\delta)$ is

$$
Z(\delta):=\{z \in \bar{D}|| g(z) \mid \leq \delta\}
$$

Of course the inclusion $Z_{g} \subset Z(\delta)$ holds.

Following B. Wick [18], we will use the following terminology:

Definition 3.7. Let $f, g \in A_{\mathbb{R}}(\bar{D})$. The function $f$ is said to be positive on real zeros of $g$ (abbreviated as $f$ is POZ of $g$ ), if $f$ has the same sign at all real zeros of $g$.

For example, consider $(f, g):=\left(z, 1-z^{2}\right) \in A_{\mathbb{R}}(\overline{\mathbb{D}})^{2}$. Then $f$ is not POZ of $g$.

3.3. Technical lemmata. In this subsection, we will prove two technical lemmata which will be used in the sequel. The first one is well-known among the workers in the field. For the sake of completeness we include a proof.

Lemma 3.8. For every function $g \in A_{\mathbb{R}}(\overline{\mathbb{D}})$, the complement $\mathbb{C} \backslash Z(\delta)$ of the level set $Z(\delta)$ is connected. Moreover, the complement $\mathbb{C} \backslash Z_{g}$ of the zero set $Z_{g}$ is also connected.

Proof. For constant functions $g$ the assertions are trivially true. So we may assume that $g$ is non constant. By the very definition $\mathbb{C} \backslash \overline{\mathbb{D}} \subset \mathbb{C} \backslash Z(\delta)$, and so if $\mathbb{C} \backslash Z(\delta)$ is not connected, there exists a bounded component $G \subset \overline{\mathbb{D}}$. Being in the complement of the level set, we must have $|g(z)| \geq \delta$ for all $z \in \partial G \subset \overline{\mathbb{D}}$. On the other hand, $|g(z)| \leq \delta$ for all $z \in \partial G \subset \overline{\mathbb{D}}$, because $\partial G \subset \partial(\mathbb{C} \backslash Z(\delta))=\partial Z(\delta) \subset Z(\delta)$. This gives $|g(z)|=\delta$ for all $z \in \partial G$, forcing $g$ to be a constant by the maximum modulus theorem, a contradiction. Hence no such bounded component of the complement of $Z(\delta)$ can exist. That $\mathbb{C} \backslash Z_{g}$ is connected follows from

$$
\mathbb{C} \backslash Z_{g}=\bigcup_{\delta>0}(\mathbb{C} \backslash Z(\delta))
$$

and the fact that $\bigcap_{\delta>0}(\mathbb{C} \backslash Z(\delta)) \neq \emptyset$ (indeed, $\mathbb{C} \backslash \overline{\mathbb{D}} \subset \mathbb{C} \backslash Z(\delta)$ for every $\delta>0$ ).

In order to facilitate handling zero sets, we prove the following result, in which we enclose the zero set by finitely many closed sets.

Lemma 3.9. Let $g \in A_{\mathbb{R}}(\overline{\mathbb{D}})$ be such that it has at least one zero in $\overline{\mathbb{D}}$, but it does not vanish identically. Then for all $\delta>0$, there exist finitely many closed sets $H_{1}, \ldots, H_{N} \subset \overline{\mathbb{D}}$, lying symmetrically with respect to the real axis, that is, $H_{j}=H_{k}^{*}$ for certain $j, k$, with the following properties:

(1) $Z_{g} \subset \cup_{j=1}^{N} H_{j} \subset Z(\delta)$.

(2) $H_{j} \cap H_{k}=\emptyset(j \neq k)$.

(3) $1^{\circ}$ If no real zero of $g$ belongs to $H_{j}$, then $H_{j} \cap \mathbb{R}=\emptyset, H_{j}$ belongs entirely to the upper (respectively lower) half plane, and $H_{j}=H_{k}^{*}$ for some $j \neq k$.

$2^{\circ}$ If at least one real zero $x_{0}$ of $g$ belongs to $H_{j}$ (that is, $x_{0} \in Z_{g} \cap H_{j} \cap \mathbb{R}$ ), then $H_{j}=H_{j}^{*}$ holds and $H_{j}$ is connected.

Proof. The zero set $Z_{g}$ is compact, hence finitely many components $K_{j}, j=1, \ldots, M$, of the relatively open set $H:=\{z \in \overline{\mathbb{D}}|| g(z) \mid<\delta\}$ will suffice to cover $Z_{g}$. Since $H$ is symmetric with respect to the real axis, its components are symmetric as well. Unfortunately, the 
closures $\overline{K_{j}}$ need not be disjoint. However, we may take the closed connected components of $\bigcup_{j=1}^{N} \overline{K_{j}}$; at most there are $M$ such components. These components are symmetric as well.

To ensure all the three assertions hold, we must eventually truncate the closed sets $\overline{K_{j}}$ :

$1^{\circ}$ If no real zero of $g$ is in $\overline{K_{j}}$, then $|g(z)| \geq \rho_{j}>0$ for all $z \in\left(\overline{K_{j}} \cap \mathbb{R}\right) \times\left(|\operatorname{Im}(z)| \leq \delta_{j}\right)$. Hence no zero of $g$ belongs to $z \in\left(\overline{K_{j}} \cap \mathbb{R}\right) \times\left(|\operatorname{Im}(z)| \leq \delta_{j}\right)$. We truncate as follows: $H_{j}:=\overline{K_{j}} \cap\left(|\operatorname{Im}(z)| \geq \delta_{j}\right)$. The closed set $K_{j}$ splits in two closed sets belonging entirely to the upper (respectively lower) half plane.

$2^{\circ}$ If at least one real zero of $g$ belongs to $\overline{K_{j}}$, then we don't truncate, that is, $H_{j}:=\overline{K_{j}}$. By symmetry we have $H_{j}=H_{j}^{*}$, and $H_{j}=\overline{K_{j}}$ is connected, because $K_{j}$ is.

By construction all the zeros of $g$ belong to exactly one closed set $H_{j}, j=1, \ldots, N$.

\section{Reducibility IN REAL SYMMETRIC ALGEBRAS ON THE DisC}

In this section we generalize the main result of B. Wick [18] to subalgebras $\mathcal{A}$ of $A_{\mathbb{R}}(\overline{\mathbb{D}})$.

In real algebras $\mathcal{A} \subset A_{\mathbb{R}}(\bar{D})$ where the corona theorem holds, the real inversion set from Theorem 3.3 is given by

$$
I_{\mathbb{R}}(f, g)=\mathbb{R} \backslash f\left(Z_{g}\right) .
$$

That the corona theorem holds for $A_{\mathbb{R}}(\bar{D})$ follows easily from the corona theorem for the complex algebra $A(\bar{D})$ by symmetrization of the solution. We refer the reader to [10] for a constructive proof (not using any Gelfand theory nor Banach algebra theory) of the corona theorem for certain subalgebras of $A(\bar{D})$ and certain domains $D$ ( including, of course, $\mathbb{D}$ ).

Theorem 4.1 (Units). Let $\mathcal{A}$ denote one of the algebras $A_{\mathbb{R}}(\overline{\mathbb{D}}), C_{\mathbb{R}}(\overline{\mathbb{D}})$ respectively. For any unit $u \in \mathcal{A}^{-1}$ either $u$ or $-u$ can be expressed as

$$
u=\exp (h)
$$

where $h \in \mathcal{A}$.

Proof. First of all we prove the theorem in case $\mathcal{A}=C_{\mathbb{R}}(\overline{\mathbb{D}})$. Choose a small closed disc $U \supset \overline{\mathbb{D}}$, so small that a continuous extension of $u$ to $U$ has no zeros in $\bar{U}$. Using a theorem of Borsuk [3, Corollary 4.33], there exists a continuous logarithm $h$ on $U \supset \overline{\mathbb{D}}$. However, this function $h$ need not be symmetric. Because the unit $u$ is symmetric we derive

$$
u(z)=\exp (h(z))=\exp \left(\left(h\left(z^{*}\right)\right)^{*}\right), \quad z \in \overline{\mathbb{D}} .
$$

Because $\overline{\mathbb{D}}$ is connected and $h$ is continuous in $\overline{\mathbb{D}}$, there exists an integer $k$ such that

$$
h(z)=\left(h\left(z^{*}\right)\right)^{*}+2 k \pi i .
$$

Restricting to the interval $[-1,1]$ gives $\operatorname{Im} h(x)=k \pi$. Since $u$ is a unit, $u(z)$ is either a positive or negative real number when $z \in[-1,1]$.

$1^{\circ}$ If $u(x)>0$, then we have $u(x)=\exp h(x)>0$, that is, the integer $k$ is even. But then $h-k \pi i$ is a symmetric continuous logarithm of $u$.

$2^{\circ}$ If $u(x)<0$, then we just look at the unit $-u$.

Hence there exists $h \in C_{\mathbb{R}}(\overline{\mathbb{D}})$ such that $u=\exp (h)$.

The remaining case $\mathcal{A}=A_{\mathbb{R}}(\overline{\mathbb{D}})$ now follows from the first case and the implicit function theorem. 
Theorem 4.2. Let $\mathcal{A}$ denote a subalgebra of $A_{\mathbb{R}}(\overline{\mathbb{D}})$ containing all real polynomials such that the corona theorem holds for $\mathcal{A}$. The following are equivalent for any unimodular pair $(f, g) \in U_{2}(\mathcal{A})$ :

(1) There exists a continuous and zero free extension $F$ of $f$ from the zero set $Z_{g}$ to $F \in C_{\mathbb{R}}(\overline{\mathbb{D}})^{-1}$.

(2) $(f, g)$ is reducible in $\mathcal{A}$, that is, there exists a unit $u \in \mathcal{A}^{-1}$ and there exists a $h \in \mathcal{A}$ such that $f+h g=u$.

Proof. The implication $(2) \Rightarrow(1)$ is obvious: indeed if there exist $h \in \mathcal{A}$ and a unit $u \in \mathcal{A}^{-1}$ such that $f+h g=u$, then $u$ serves as the desired zero free extension of $f$ from the zero set $Z_{g}$ to $u \in C_{\mathbb{R}}(\overline{\mathbb{D}})^{-1}$.

$(1) \Rightarrow(2)$ : Using Theorem 4.1 for units in $C_{\mathbb{R}}(\overline{\mathbb{D}})$, we write either $F$ or $-F$ as

$$
F=\exp (K)
$$

where $K \in C_{\mathbb{R}}(\overline{\mathbb{D}})$. For either $f$ or $-f$ this gives

$$
f(z)=\exp (K(z)) \quad\left(z \in Z_{g}\right),
$$

and so $f$ (respectively $-f$ ) is in fact an exponential on the zero set $Z_{g}$.

If $g$ is the zero function then $f$ must be invertible in $\mathcal{A}$, because the pair $(f, 0)$ was assumed to be unimodular. So $(f, 0)$ is reducible in $\mathcal{A}$, and the unit $u$ is just the function $f$. Hence we may assume that $g$ is not the zero function, and so the interior of the zero set $Z_{g}$ is empty by the identity theorem. By Lemma 3.8 the complement $\mathbb{C} \backslash Z_{g}$ of the zero set is connected. Using Mergelyan's theorem there exist polynomials $q_{n}$ converging uniformly on $Z_{g}$ to $K$. Because $K$ is symmetric, we can also approximate by the symmetrization of $q_{n}$, that is, the real polynomials $\widetilde{q}_{n}$ given by

$$
\widetilde{q}_{n}(z):=\frac{q_{n}(z)+\left(q_{n}\left(z^{*}\right)\right)^{*}}{2}
$$

converge uniformly to $K$ too. Pick a real polynomial $q$ such that

$$
\operatorname{Re}(f(z) \exp (-q(z)))>1 / 2 \quad\left(z \in Z_{g}\right) .
$$

Using the corona theorem, all pairs $(f \exp (-q)-\lambda, g)$ are unimodular for $\lambda \in(-\infty, 1 / 2)$. For the real inversion set from Theorem 3.3 this gives the inclusion

$$
I_{\mathbb{R}}(f \exp (-q), g)=\mathbb{R} \backslash(f \exp (-q))\left(Z_{g}\right) \supset(-\infty, 1 / 2) .
$$

Again Theorem 3.3 shows that $(f \exp (-q), g)$ is reducible in $A_{\mathbb{R}}(\overline{\mathbb{D}})$, hence $(f, g)$, that is, there exists a unit $U \in A_{\mathbb{R}}(\overline{\mathbb{D}})^{-1}$ and $H \in A_{\mathbb{R}}(\overline{\mathbb{D}})$ such that

$$
f+H g=U \text {. }
$$

$U$ being a unit, we must have

$$
|U(z)|>\delta>0 \quad(z \in \overline{\mathbb{D}}) .
$$

Now the real polynomials are dense in $A_{\mathbb{R}}(\overline{\mathbb{D}})$. Take real polynomials $h \in \mathcal{A}$ near $H$ such that $|U(z)-(H(z)-h(z)) g(z)|>\delta / 2>0(z \in \overline{\mathbb{D}})$. We conclude that

$$
f+h g=U-(H-h) g
$$

belongs to the algebra $\mathcal{A}$ and has no zeros in $\overline{\mathbb{D}}$ and so it is invertible, proving the reducibility of $(f, g)$ in $\mathcal{A}$. 
Theorem 4.3. Let $\mathcal{A}$ denote a subalgebra of $A_{\mathbb{R}}(\overline{\mathbb{D}})$ containing all real polynomials such that the corona theorem holds for $\mathcal{A}$. The following assertions are equivalent for any unimodular $\operatorname{pair}(f, g) \in \mathcal{A}^{2}$ :

(1) $(f, g)$ is reducible in $\mathcal{A}$.

(2) $f$ is POZ of $g$.

Proof. $(1) \Rightarrow(2)$ : Suppose that $(f, g)$ is reducible in $\mathcal{A}$. Then there exist $h, u \in \mathcal{A}, u \in \mathcal{A}^{-1}$ such that

$$
f+h \cdot g=u \text {. }
$$

By Theorem 4.1 either $u$ or $-u$ can be written as

$$
u=\exp (k)
$$

for a function $k \in A_{\mathbb{R}}(\overline{\mathbb{D}})$. We arrive at $f+h \cdot g=\exp (k)$, respectively the same equation with $-\exp (k)$ instead of $\exp (k)$. Hence the function $f$ is POZ of $g$.

$(2) \Rightarrow(1)$ : Now assume that $f$ is POZ of $g$. If $g$ has no zero at all in $\overline{\mathbb{D}}$, then we have $|g(z)| \geq \rho>0$ for all $z \in \overline{\mathbb{D}}$. But then $M:=\left(1+\|f\|_{\infty}\right) / \rho$ gives

$$
|f(z)+M g(z)|>1+\|f\|_{\infty}-\|f\|_{\infty}=1 \text {, }
$$

and so $f+M g$ is invertible in $A$; hence the pair $(f, g)$ is reducible. We may also assume that $g$ is not the zero function. Otherwise $f$ itself is invertible and again the pair $(f, g)$ would be reducible. So our assumption is: $g$ has at least one zero in $\overline{\mathbb{D}}$ but is not identically zero. In order to use Theorem 4.2, we must show that there exists a continuous, zero free extension $F$ of $f$ from the zero set $Z_{g}$ to $\overline{\mathbb{D}}$.

Because $(f, g)$ is unimodular there exists $\delta>0$ such that $|f(z)|+|g(z)| \geq \delta$ for all $z \in \overline{\mathbb{D}}$. By Lemma 3.9 (with $\delta / 2$ instead of $\delta$ ), there exist finitely many pairwise disjoint closed sets $H_{1}, \ldots, H_{N} \subset \overline{\mathbb{D}}$ lying symmetrically with respect to the real axis, such that: $Z_{g} \subset \cup_{j=1}^{N} H_{j}$ and $|g(z)| \leq \delta / 2$ holds there. Hence $|f(z)| \geq \delta / 2$ in the union of this sets. Moreover, we have a continuous logarithm of $f$ on $Z(\delta / 2)$ : To prove this we quote a theorem of Borsuk, see [3, Corollary 4.33]: Every continuous, zero-free function on $Z(\delta)$ has a continuous logarithm on $Z(\delta)$ if and only if $\mathbb{C} \backslash Z(\delta)$ is connected. This is the case by Lemma 3.8. In particular, there exist functions $l_{j}$, continuous in the closed sets $H_{j} \subset Z(\delta)$, such that

$$
f(z)=\exp \left(l_{j}(z)\right), \quad z \in H_{j}, \quad j=1, \ldots, N .
$$

By assertion (3) of Lemma 3.9, if no real zero of $g$ belongs to $H_{j}$ then $H_{j} \cap \mathbb{R}=\emptyset$. Moreover, $H_{j}$ belongs entirely to the upper (respectively lower) half plane. The desired logarithm is very easy to obtain for these sets, because they don't intersect the real line. By symmetry we have $H_{j}=H_{k}^{*}$ for some $j \neq k$. So we may redefine $l_{j}(z)=\left(l_{k}\left(z^{*}\right)\right)^{*}$.

Thus only the case of a real zero $x_{0}$ of $g$ belonging to $H_{j}$ remains to be discussed. In this case $H_{j}$ is connected. Since $f$ is $\mathrm{POZ}$ of $g$, we may assume that $f\left(x_{0}\right)>0$ holds for all real zeros of $g$. Because $f$ is real symmetric we derive

$$
f(z)=\exp \left(l_{j}(z)\right)=\exp \left(\left(l_{j}\left(z^{*}\right)\right)^{*}\right) \quad\left(z \in H_{j}=H_{j}^{*}\right) .
$$

Since $H_{j}$ is connected and $l_{j}$ is continuous in $H_{j}$, there exists an integer $k$ such that

$$
l_{j}(z)=\left(l_{j}\left(z^{*}\right)\right)^{*}+2 k \pi i .
$$

Restricting to the real zero $x_{0} \in H_{j} \cap \mathbb{R}$ of $g$ gives $\operatorname{Im} l_{j}\left(x_{0}\right)=k \pi$. As $f\left(x_{0}\right)=\exp \left(l_{j}\left(x_{0}\right)\right)>0$, the integer $k$ must be even. Now $l_{j}-k \pi i$ is the desired symmetric logarithm of $f$ on $H_{j}=H_{j}^{*}$. 
By Tietze's Theorem we can find a continuous function $l$ on $\overline{\mathbb{D}}$ such that

$$
l(z)=l_{j}(z) \quad z \in H_{j}, \quad j=1, \ldots, N .
$$

The desired logarithm is now given by symmetrization in $\overline{\mathbb{D}}$ :

$$
L(z):=\frac{l(z)+\left(l\left(z^{*}\right)\right)^{*}}{2} .
$$

Recall that we either have $l_{j}(z)=\left(l_{k}\left(z^{*}\right)\right)^{*}$ for $H_{j}=H_{k}^{*}, j \neq k$, or else $l_{j}(z)=\left(l_{j}\left(z^{*}\right)\right)^{*}$ and $H_{j}=H_{j}^{*}$. We end up with $F=\exp (L)$, where we have $\exp (L(z))=f(z)\left(z \in Z_{g}\right)$.

$$
\text { 5. Units in } A_{\mathbb{R}}(\bar{D}) \text { AND } C_{\mathbb{R}}(\bar{D})
$$

We recall the notation of the winding number $n(\Gamma ; z)$ from [3, Definition 4.2]:

Let $\Gamma$ denote a closed loop given by a continuous parametrization $t \mapsto \zeta(t)(a \leq t \leq b)$, and $z$ denote a point outside $\Gamma$. Then there exists a continuous logarithm $h$ of $\zeta(t)-z$, that is

$$
\zeta(t)-z=\exp (h(t)), \quad a \leq t \leq b .
$$

The winding number $n(\Gamma ; z)$ is defined to be

$$
n(\Gamma ; z):=\frac{h(b)-h(a)}{2 \pi i}=\frac{\phi(b)-\phi(a)}{2 \pi},
$$

where $\phi$ denotes the imaginary part of the $\operatorname{logarithm} h$. From this definition the following facts are easily seen:

(F1) Let $f, g$ denote zero free continuous functions near the closed loop $\Gamma$. Then we can form the closed loops $f(\Gamma), g(\Gamma),(f \cdot g)(\Gamma)$ by their parametrizations $f(\zeta(t)), g(\zeta(t))$, $(f \cdot g)(\zeta(t))$, respectively. Since $f$ and $g$ never vanish on $\Gamma$, we conclude

$$
n((f \cdot g)(\Gamma) ; 0)=n(f(\Gamma) ; 0)+n(g(\Gamma) ; 0) .
$$

Also $n(\exp (f)(\Gamma) ; 0)=0$.

(F2) The curves $\Gamma_{r+m+j}$ are the reflection of $\Gamma_{r+j}$ with reversed orientation, and so

$$
n\left(f\left(\Gamma_{r+m+j}\right) ; 0\right)=n\left(f\left(\Gamma_{r+j}\right) ; 0\right)
$$

holds for all continuous symmetric functions, zero free near $\Gamma_{r+j}$.

Theorem 5.1 (Product theorem for units). Let the notations be as in Notation 3.4. Let $\mathcal{A}$ denote one of the algebras $A_{\mathbb{R}}(\bar{D}), C_{\mathbb{R}}(\bar{D})$. For any unit $u \in \mathcal{A}^{-1}$ there exist integers $n_{1}, \ldots, n_{r}, n_{r+1}, \ldots, n_{r+m}$ and a function $h \in \mathcal{A}$ such that the following structure theorem holds: Either $u$ or $-u$ can be factored as

$$
u=p \cdot \exp (h)
$$

where $p \in \mathcal{A}^{-1}, h \in \mathcal{A}$ and the unit $p$ is given by

$$
p(z)=\prod_{j=1}^{r}\left(z-x_{j}\right)^{n_{j}} \cdot \prod_{j=1}^{m}\left(z^{2}-2\left(\operatorname{Re}\left(z_{j}\right)\right) z+\left|z_{j}\right|^{2}\right)^{n_{r+j}} .
$$

Proof. Choose a small compact neighborhood $U \supset \bar{D}$, so small that a continuous extension of $u$ to $U$ has no zeros in $U$. Each hole of $U$ belongs to exactly one hole of $D$. The factorization for $u$ in $U$ follows then from [3, Theorem 4.59]. To be precise:

$$
u=p \cdot \exp (h),
$$


where $p \in \mathcal{A}^{-1}, h$ is analytic in $U \supset \bar{D}$, and the unit $p$ is given by

$$
p(z)=\prod_{j=1}^{r}\left(z-x_{j}\right)^{n_{j}} \prod_{j=1}^{m}\left(z-z_{j}\right)^{n_{r+j}} \prod_{j=1}^{m}\left(z-z_{j}^{*}\right)^{n_{r+m+j}} .
$$

With these products and the facts above we can compute the integers $n_{k}(k=1, \ldots, r+2 m)$ :

$n\left(u\left(\Gamma_{k}\right) ; 0\right)=\sum_{j=1}^{r} n_{j} \cdot n\left(\Gamma_{k} ; x_{j}\right)+\sum_{j=1}^{m} n_{r+j} \cdot n\left(\Gamma_{k} ; z_{j}\right)+\sum_{j=1}^{m} n_{r+m+j} \cdot n\left(\Gamma_{k} ; z_{j}^{*}\right)+n\left(\exp (h)\left(\Gamma_{k}\right) ; 0\right)$,

that is, $n\left(u\left(\Gamma_{k}\right) ; 0\right)=n_{k}(k=1, \ldots, r+2 m)$. Recall that the curves $\Gamma_{r+m+j}$ are the reflection of $\Gamma_{r+j}$. Observe that reflection has reversed orientation. Using the fact F2 and the symmetric choice of our points, we derive

$$
n_{r+m+j}=n\left(u\left(\Gamma_{r+m+j}\right) ; 0\right)=n\left(u\left(\Gamma_{r+j}\right) ; 0\right)=n_{r+j} \quad(j=1, \ldots, m) .
$$

We conclude that

$$
\left(z-z_{j}^{*}\right)^{n_{r+m+j}} \cdot\left(z-z_{j}\right)^{n_{r+j}}=\left(z^{2}-2 \cdot \operatorname{Re}\left(z_{j}\right) \cdot z+\left|z_{j}\right|^{2}\right)^{n_{r+j}} \quad(j=1, \ldots, m) .
$$

This proves the product representation. We now show that the logarithm $h$ can be chosen to be symmetric. Using the symmetry of the functions $u$ and $p$, we conclude that

$$
\exp \left(h\left(z^{*}\right)^{*}\right)=\exp (h(z))
$$

holds in the connected set $\bar{D}$. Thus there is an integer $k$ such that $h(z)=\left(h\left(z^{*}\right)\right)^{*}+2 k \pi i$. Hence $\operatorname{Im}(h(x))=k \pi(x \in \mathbb{R} \cap \bar{D})$. Take a point $x_{0} \in \mathbb{R} \cap \bar{D}$, such that $x_{0}>\max \left\{x_{1}, \ldots, x_{r}\right\}$. Then $p\left(x_{0}\right)>0$, and we consider the two cases:

$1^{\circ}$ If $u\left(x_{0}\right)>0$, then we have $\exp h\left(x_{0}\right)=u\left(x_{0}\right) / p\left(x_{0}\right)>0$. Since $\operatorname{Im}\left(h\left(x_{0}\right)\right)=k \pi$ it follows that $k$ is even. But then $h-k \pi i$ is a symmetric logarithm of $u / p$.

$2^{\circ}$ If $u\left(x_{0}\right)<0$, then we just look at the unit $-u$.

\section{Reducibility in ALGEBras of ReAl Symmetric FunCtions}

In this section we generalize our technical lemmata to the case of certain finitely connected domains.

Lemma 6.1. Let $D$ denote a domain as described in Notation 3.4. For every non constant function $g \in A_{\mathbb{R}}(\bar{D})$, the complement $\mathbb{C} \backslash Z(\delta)$ of the level set $Z(\delta)$ is connected for all sufficiently small $\delta>0$. Moreover, the complement $\mathbb{C} \backslash Z_{g}$ of the zero set $Z_{g}$ is also connected.

Proof. We will first prove that the complement $\mathbb{C} \backslash Z_{g}$ of the zero set is connected. If $g$ is not identically zero, then by [15, Theorem 3.1], the zero set $Z_{g} \subset \bar{D}$ is totally disconnected, and so its covering dimension is zero. Hence its open complement $\mathbb{C} \backslash Z_{g}$ is connected, see [8, Theorem IV.4].

Now we prove that $\mathbb{C} \backslash Z(\delta)$ is connected. We connect each hole $C_{j}$ by pairwise disjoint crosscuts $Q_{j} \subset \bar{D}, j=1, \ldots, n$, connecting $\Gamma_{j}$ to $\Gamma_{j+1}$ such that $g(z) \neq 0$ for all $z \in \bigcup_{j=1}^{n} Q_{j}$. (This can best be done mapping $\bar{D}$ homeomorphically onto the closure of a circular domain, see for example [11]. Note that even in this situation, the zero set remains totally disconnected.) 
Assume that $\delta$ is sufficiently small, that is $\delta<\delta_{0} / 2$, where

$$
\delta_{0}:=\min \left\{|g(z)|, z \in \bigcup_{j=1}^{n} Q_{j}\right\} .
$$

From Topology we know that $D \backslash \bigcup_{j=1}^{n}\left(Q_{j} \cup C_{j}\right)$ is a simply connected domain. The restriction to the choice $\delta$ gives

$$
Z(\delta) \subset \bar{D} \backslash \bigcup_{j=1}^{n}\left(Q_{j} \cup C_{j}\right) .
$$

If $\mathbb{C} \backslash Z(\delta)$ is not connected, then there exists a bounded component $G$. In contrast to the simply connected case $\mathbb{D}$, we may have two cases:

$1^{\circ} G \subset \bar{D}$ or

$2^{\circ} G \cap(\mathbb{C} \backslash \bar{D}) \neq \emptyset$, that is, there exists $z_{0} \in G$ belonging to a hole, say $C$ : so $z_{0} \in C$. But then the hole $C$ is contained in $G$. Using our cross-cuts we can find a path in $\mathbb{C} \backslash Z(\delta)$ connecting $C$ to the outer boundary $\Gamma_{n+1}$ of $D$ and beyond. So the starting point of this path belongs to $\bar{G}$, whereas the endpoint does not. Hence there exists a boundary point $w \in \partial G$ belonging to $\bigcup_{j=1}^{n} Q_{j} \subset \bar{D}$. Since $\partial G \subset \partial(\mathbb{C} \backslash Z(\delta))=\partial Z(\delta) \subset Z(\delta)$ we must have $|g(w)| \leq \delta$, contradicting the choice of $\delta<\frac{\delta_{0}}{2}=\frac{1}{2} \min \{|g(z)|, \quad z \in$ $\left.\bigcup_{j=1}^{n} Q_{j}\right\}$.

Thus only the first case $G \subset \bar{D}$ remains to be dealt with. But this is done exactly as in the proof of Lemma 3.8. Hence no such bounded component of the complement of $Z(\delta)$ can exist.

Lemma 6.2. Let $D$ denote a domain as described in Notation 3.4 and let $g \in A_{\mathbb{R}}(\bar{D})$ be such that it has at least one zero in $\bar{D}$, but it does not vanish identically. Then for all $\delta>0$, there exist finitely many closed sets $H_{1}, \ldots, H_{N} \subset \bar{D}$, lying symmetrically with respect to the real axis, that is, $H_{j}=H_{k}^{*}$ for certain $j, k$, with the following properties:

(1) $Z_{g} \subset \cup_{j=1}^{N} H_{j} \subset Z(\delta)$.

(2) $H_{j} \cap H_{k}=\emptyset(j \neq k)$.

(3) $1^{\circ}$ If no real zero of $g$ belongs to $H_{j}$, then $H_{j} \cap \mathbb{R}=\emptyset, H_{j}$ belongs entirely to the upper (respectively lower) half plane, and $H_{j}=H_{k}^{*}$ for some $j \neq k$.

$2^{\circ}$ If at least one real zero $x_{0}$ of $g$ belongs to $H_{j}$ (that is, $x_{0} \in Z_{g} \cap H_{j} \cap \mathbb{R}$ ), then $H_{j}=H_{j}^{*}$ holds and $H_{j}$ is connected.

Proof. The proof proceeds exactly as in the proof of Lemma 3.9. We just replace the use of Lemma 3.8 by Lemma 6.1 .

Theorem 6.3. Let the notations be as in 3.4 above. Let $\mathcal{A}$ denote a subalgebra of $A_{\mathbb{R}}(\bar{D})$ containing all real polynomials such that the corona theorem holds for $\mathcal{A}$. Let $(f, g) \in \mathcal{A}^{2}$ be a unimodular pair. Then the following assertions are equivalent:

(1) There exists a continuous and zero free extension $F$ of $f$ from the zero set $Z_{g}$ to $F \in C_{\mathbb{R}}(\bar{D})^{-1}$.

(2) $(f, g)$ is reducible in $\mathcal{A}$, that is, there exists a unit $u \in \mathcal{A}^{-1}$ and $h \in \mathcal{A}$ such that $f+h g=u$.

Proof. The implication $(2) \Rightarrow(1)$ is obvious: indeed if there exist $h \in \mathcal{A}$ and a unit $u \in \mathcal{A}^{-1}$ such that $f+h g=u$, then $u$ serves as the desired zero free extension of $f$ from the zero set $Z_{g}$ to $u \in C_{\mathbb{R}}(\bar{D})^{-1}$. 
$(1) \Rightarrow(2)$ : Using the factorization theorem 5.1 for units in $C_{\mathbb{R}}(\bar{D})$, we can factor either $F$ or $-F$ as

$$
F=p \cdot \exp (K)
$$

where $p \in \mathcal{A}^{-1}$ (because $\mathcal{A}$ contains all polynomials and the corona theorem holds) and $K \in C_{\mathbb{R}}(\bar{D})$. For either $f$ or $-f$ this gives

$$
\frac{f(z)}{p(z)}=\exp (K(z)) \quad\left(z \in Z_{g}\right)
$$

and so the fraction is in fact an exponential on the zero set $Z_{g}$. The rest of the proof is now entirely analogous to the corresponding case in the proof of Theorem 4.2.

Recall the Notation 3.4: Let $D$ denote a symmetric domain in $\mathbb{C}$ with $n$ holes. From these $n$ holes, bounded by pairwise disjoint Jordan curves, we have $r$ holes intersecting $\mathbb{R}$ and $2 m$ which do not intersect $\mathbb{R}$. Here $n=r+2 m$.

Let $C_{j}$ be a hole of $D(j=1, \ldots, r, r+1, \ldots, r+2 m)$. Choose for each $j \in\{1, \ldots, r\}$ a point $x_{j} \in \mathbb{R} \cap C_{j}$, and for $j \in\{r+1, \cdots r+m\}$ let $z_{j} \in C_{j}$.

Finally let $S=\left\{x_{1}, \ldots, x_{r}, z_{1}, \ldots, z_{m}, z_{1}^{*}, \ldots, z_{m}^{*}\right\}$. See Figure 1. For such domains we associate the following family of $2^{r}$ polynomials:

$$
\mathcal{P}:=\left\{p(z):=\prod_{j=1}^{r}\left(z-x_{j}\right)^{m_{j}} \mid m_{j} \in\{0,1\}\right\} .
$$

As an example, consider an annulus with center at the origin, and let us choose $x_{1}=0$. Then $\mathcal{P}$ has only two polynomials, namely $p_{1}(z):=1$ and $p_{2}(z):=z$.

In [18], Brett Wick showed that a unimodular pair $(f, g)$ in $A_{\mathbb{R}}(\overline{\mathbb{D}})^{2}$ is reducible in $A_{\mathbb{R}}(\overline{\mathbb{D}})$ if and only if $f$ is POZ of $g$. We generalize this result from the case of $\mathbb{D}$ to our domains $D$.

Theorem 6.4. As in Notation 3.4, let $D$ denote a symmetric domain with $n$ holes, bounded by pairwise disjoint Jordan curves, and let $\mathcal{P}$ denote the associated family of $2^{r}$ polynomials (1). Let $\mathcal{A}$ denote a subalgebra of $A_{\mathbb{R}}(\bar{D})$ containing all real polynomials such that the corona theorem holds for $\mathcal{A}$. The following assertions are equivalent for a unimodular pair $(f, g) \in \mathcal{A}^{2}$ :

(1) $(f, g)$ is reducible in $\mathcal{A}$.

(2) For at least one polynomial $p \in \mathcal{P}$ the product $p \cdot f$ is $P O Z$ of $g$.

Proof. $(1) \Rightarrow(2)$ : Suppose that $(f, g)$ is reducible in $\mathcal{A}$. Then there exist $h, u \in \mathcal{A}, u \in \mathcal{A}^{-1}$ such that

$$
f+h \cdot g=u .
$$

By the factorization theorem for units (Theorem 5.1) in $A_{\mathbb{R}}(\bar{D})$, there exists an invertible polynomial $P \in \mathcal{A}$ such that either $u$ or $-u$ can be factored as

$$
u=P \cdot \exp (k),
$$

for a function $k \in A_{\mathbb{R}}(\bar{D})$. We arrive at $f+h \cdot g=P \cdot \exp (k)$, respectively the same equation with $-P$ instead of $P$. Hence the function $P \cdot f$ is POZ of $g$. But the sign of $P(x)$ depends only on the exponents modulo 2 of its linear factors $x-x_{j}$. So we can find a polynomial $p \in \mathcal{P}$ such that $p \cdot f$ is POZ of $g$.

$(2) \Rightarrow(1)$ : Now assume that for a polynomial $p \in \mathcal{P}$ the product $p \cdot f$ is POZ of $g$. Since $p \in \mathcal{A}^{-1}$, it is enough to show that the corona pair $(\widetilde{f}, g)$ is reducible, where $\widetilde{f}:=f / p$. The rest of the proof is entirely analogous to the corresponding case in the proof of Theorem 4.3. 
Remark 6.5. The family $\mathcal{P}$ obviously depends on the choice of points in the associated set $S$, hence so does the second assertion in Theorem 6.4, whereas the question of reducibility is independent of the particular choice of $S$. This mystery can be cleared by Eilenberg's theorem [3, Exercise 4.36, p. 100]:

For any two points $a, b$ belonging to the same hole of $D$ there exists a continuous logarithm $\log \frac{z-a}{z-b}$ which we think of as extended continuously to $\mathbb{C}$. Thus the product representation of the unit in the proof of Theorem 6.4 won't change much as long as both points belong to the same hole.

The proof of Theorem 2.1 can be generalized to the real Banach algebras $A_{\mathbb{R}}(\bar{D})$, where $D$ is as in Notation 3.4. By Mergelyan's theorem, (real) rational functions are dense in this Banach algebra, and so the proof alters only slightly. So we conclude that the Bass stable rank of these algebras is less than or equal 2. However, our domains $D$ contain an open interval $(a, b) \subset \mathbb{R}$. Consider the unimodular pair $\left(z-\frac{a+b}{2},(z-a)(z-b)\right)$. The necessary condition that $f$ is POZ of $g$ is violated, and so this pair is not reducible, hence the stable rank of $A_{\mathbb{R}}(\bar{D})$ is 2 . Are there situations where the stable rank of the real algebras is one? Obviously we must allow open sets instead of domains, since there are no symmetric domains such that $D \cap \mathbb{R}=\emptyset$.

Question: For which bounded symmetric open sets $D \subset \mathbb{C}$ do we have bsr $A_{\mathbb{R}}(\bar{D})=1$ ?

If the stable rank of $A_{\mathbb{R}}(\bar{D})$ is 1 , then the open set $D$ necessarily must fulfill the following requirement: $\bar{D} \cap \mathbb{R}$ is either empty or totally disconnected.

If $\bar{D} \cap \mathbb{R}$ is not empty and not totally disconnected, then it contains an open interval $(a, b) \subset \mathbb{R}$. The unimodular pair $\left(z-\frac{a+b}{2},(z-a)(z-b)\right)$ is again not reducible by the intermediate value theorem.

Theorem 6.6. Let $D \subset \mathbb{C}$ be a bounded symmetric open set such that $D^{+}:=D \cap\{\operatorname{Im}(z)>0\}$ is a finitely connected domain and $\bar{D} \cap \mathbb{R}$ is either empty or a totally disconnected set of linear measure zero. Then the Bass stable rank of $A_{\mathbb{R}}(\bar{D})$ is 1 .

Proof. Using [5, Theorem 3.11], we see that the complex Banach algebra $A\left(\overline{D^{+}}\right)$has Bass stable rank one, because by Mergelyan's theorem the rational functions with poles off $\overline{D^{+}}$ are dense in $A\left(\overline{D^{+}}\right)$. Again the corona theorem holds in $A(\bar{D})$ by Arens's theorem, hence in $A_{\mathbb{R}}(\bar{D})$, see for example [6, Theorem 1.9, p. 31]. Let $(f, g)$ be a unimodular pair in $A_{\mathbb{R}}(\bar{D})$. If $\bar{D} \cap \mathbb{R}=\emptyset$, we proceed as follows: the unimodular pair $(f, g)$ is reducible in $A\left(\overline{D^{+}}\right)$, that is, there exist $u, h \in A\left(\overline{D^{+}}\right), u$ invertible in $A\left(\overline{D^{+}}\right)$such that $f+h g=u$. Now we reflect $u, h$ to the lower half plane. Since $\bar{D} \cap \mathbb{R}=\emptyset$ this reflection is well-defined, hence $(f, g)$ is reducible in $A_{\mathbb{R}}(\bar{D})$.

So we may assume that $\bar{D} \cap \mathbb{R}$ is not empty and is a totally disconnected set of linear measure zero.

$1^{\circ} f(x) \neq 0$ for all $x \in \bar{D} \cap \mathbb{R}$.

Take a peak-function $p$ in the upper half plane for the totally disconnected set $\bar{D} \cap \mathbb{R}$ of linear measure zero. The existence of such a peak function follows from Rudin's theorem (see for instance [7, p. 81]) by conformally mapping the unit disc onto the upper half plane. Then the function $q:=1-p \in A\left(\overline{D^{+}}\right)$vanishes at $z_{0}$ if and only if $z_{0} \in \bar{D} \cap \mathbb{R}$. The corona theorem for $A\left(\overline{D^{+}}\right)$and $1^{\circ}$ now implies that the pair $(f, q \cdot g)$ is unimodular in $A\left(\overline{D^{+}}\right)$, and so it is reducible in $A\left(\overline{D^{+}}\right)$. Thus there 
exists $h \in A\left(\overline{D^{+}}\right)$such that $f+h \cdot(q g)$ has no zeros in $\overline{D^{+}}$. We define the function $H \in A_{\mathbb{R}}(\bar{D})$ by reflection: $H(z):=h(z) q(z)$ in case $z \in \overline{D^{+}}$and $H(z):=\left(h\left(z^{*}\right) q\left(z^{*}\right)\right)^{*}$ in case $z \in \bar{D} \backslash D^{+}$. Since $q$ vanishes identically on $\bar{D} \cap \mathbb{R}$ this reflection is well-defined. We conclude that $f+H \cdot g$ has no zeros in $\bar{D}$ and so it is invertible by the corona theorem. Therefore the pair $(f, g)$ is reducible in $A_{\mathbb{R}}(\bar{D})$.

$2^{\circ} f(x)=0$ for some $x \in \bar{D} \cap \mathbb{R}$.

We approximate $f$ uniformly by real rational functions $f_{n}$ on $\bar{D}$, and again use Mergelyan's theorem and symmetry. We perturb the finitely many zeros of $f_{n}$ slightly (respecting symmetry) such that $f_{n}$ has no zeros in $\bar{D} \cap \mathbb{R}$. Using $1^{\circ}$ we see that the pairs $\left(f_{n}, g\right)$ are reducible in $A_{\mathbb{R}}(\bar{D})$, and so $(f, g)$ is reducible in $A_{\mathbb{R}}(\bar{D})$, see for example [5, Lemma 3.7].

\section{REFERENCES}

[1] L.V. Ahlfors, Complex Analysis, 3rd Edition (McGraw-Hill, New York, 1979).

[2] H. Bass, K-theory and stable algebra, Publications Mathématiques de L'I.H.É.S., 22, 5-60 (1964).

[3] R.B. Burckel, An Introduction to Classical Complex Analysis, Vol. 1 (Birkhäuser, New York-London, 1979).

[4] G. Corach and A.R. Larotonda, Stable range in Banach algebras, Journal of Pure and Applied Algebra, 32, 289-300 (1984).

[5] G. Corach and F.D. Suárez, Stable range in holomorphic function algebras, Illinois Journal of Mathematics, 29, 627-639 (1985).

[6] T.W. Gamelin, Uniform Algebras (Prentice-Hall, N.J., 1969).

[7] K. Hoffman, Banach Spaces of Analytic Functions (Dover, New York, 1962).

[8] W. Hurewicz and H.W. Wallman, Dimension Theory (Princeton University Press, Princeton, N.J., 1941).

[9] P.W. Jones, D. Marshall and T.Wolff, Stable rank of the disc algebra, Proceeding of the American Mathematical Society, no. 4, 96, 603-604 (1986).

[10] R. Mortini and R. Rupp, A constructive proof of the nullstellensatz for subalgebras of $A(K)$, Séminaire de mathématique de Luxembourg, Trav. Math., 3, 45-49 (1991).

[11] Chr. Pommerenke, Univalent Functions (Vandenhoeck and Ruprecht, Göttingen, 1975).

[12] A. Quadrat, On a general structure of the stabilizing controllers based on stable range, SIAM Journal on Control and Optimization, no. 6, 42, 2264-2285 (2004).

[13] M.A. Rieffel, Dimension and stable rank in the K-theory of $C^{*}$-algebras, Proceedings of the London Mathematical Society, no.2, 46, 301-333 (1983).

[14] R. Rupp, Stable rank in Banach algebras, Function spaces, Lecture Notes in Pure and Applied Mathematics, 136, 357-365 (Dekker, New York, 1992).

[15] R. Rupp, Stable rank of subalgebras of the disc algebra, Proceedings of the American Mathematical Society, 108, 137-142 (1990).

[16] R. Rupp, Stable rank and the $\bar{\partial}$-equation, Canadian Mathematical Bulletin, 34, 113-118 (1991).

[17] M. Vidyasagar, Control System Synthesis: A Factorization Approach, MIT Press Series in Signal Processing, Optimization, and Control, 7 (MIT Press, Cambridge, MA, 1985).

[18] B. Wick, A note about stabilization in the real disc algebra, To appear in Mathematische Nachrichten (2007).

E-mail address: rudolf.rupp@fh-nuernberg.de

Fachbereich Allgemeinwissenschaften, Georg-Simon-Ohm-Fachhochschule Nürnberg, KesslerPlatz 12, D-90489 Nürnberg, Germany.

E-mail address: A.J.Sasane@lse.ac.uk

Mathematics Department, London School of Economics, Houghton Street, London WC2A 2AE, United Kingdom. 